\title{
A spectroscopic survey for $\lambda$ Bootis stars
}

\section{Strategy, techniques and first results ${ }^{\star}$}

\author{
E. Paunzen ${ }^{1}$ and R.O. Gray ${ }^{2}$ \\ 1 Institut für Astronomie der Universität Wien, Türkenschanzstr. 17, A-1180 Wien, Austria \\ e-mail: paunzen@astro.ast.univie.ac.at \\ 2 Department of Physics and Astronomy, Appalachian State University, Boone, North Carolina 28608, U.S.A. \\ e-mail: grayro@conrad.appstate.edu
}

Received December 9, 1996; accepted April 3, 1997

\begin{abstract}
In recent years, the chemically peculiar (CP) stars of the upper main sequence have become a fruitful field for the testing of astrophysical theories. Processes such as diffusion, convection and mass loss have been developed theoretically and introduced into models. The group of $\lambda$ Bootis stars, however, is remarkable among the chemically peculiar stars as they are nonmagnetic, Population I, A to F-type dwarfs which show significant underabundances of metals (except for C, N, O and S). Unfortunately, the small number of confirmed members of the $\lambda$ Bootis class makes a sound statistical analysis of their properties impossible. Thus, it is still difficult to decide between the two theories - mass loss with diffusion and the accretion theory - which have been proposed to explain the origin of these stars.

We therefore have started a spectroscopic survey to find new $\lambda$ Bootis stars in the field as well as in open clusters and associations. The presence of $\lambda$ Bootis stars in open clusters and associations would permit a determination of the ages of these stars, and thus would yield an important test for distinguishing between the two theories. In this paper we describe the selection of candidates using photometric criteria, the basic requirements, spectroscopic follow-up observations and results from the first three observing runs. Special care was taken to avoid misclassification of our programme stars (e.g. $\lambda$ Bootis stars are often confused with intermediate Population II, Heweak or high $v \sin i$ stars), using a refined MK system. The discovery of at least six new $\lambda$ Bootis stars (including three in the Orion OB1 association) shows the efficacy of our selection criteria.
\end{abstract}

Send offprint requests to: E. Paunzen

* Based on observations obtained at the Observatoire de Haute-Provence and the Dark Sky Observatory.
Key words: surveys - stars $-\lambda$ Bootis - stars: chemically peculiar — stars: early type; stars: fundamental parameters

\section{Introduction}

For many years the nature of the $\lambda$ Bootis stars has been controversial. About $20 \%$ of all Population I A-type stars show abundance peculiarities. Most of these CP stars are characterized by strong overabundances of iron peak elements (Jaschek \& Jaschek 1995). In contrast, the $\lambda$ Bootis stars are metal poor, Populaton I, A and early F-type stars. Unfortunately, these astrophysically interesting stars are quite rare; the most recent list of confirmed members (Gray \& Corbally 1993) had only 20 members. A recent paper (Paunzen et al. 1997) attempted to expand this number by a critical assessment of the literature resulting in a total of 45 members and candidate members. This small sample of stars still makes a sound statistical analysis difficult. Therefore we have started a spectroscopic survey for new members in the galactic field, open clusters and associations. The discovery of $\lambda$ Bootis stars in open clusters and associations is especially important because of the possibility of age determination, thus providing the means to distinguish between the two proposed theories - the diffusion/mass loss theory (Michaud \& Charland 1986) and the accretion theory (Venn \& Lambert 1990). Candidate $\lambda$ Bootis stars were discovered in the Orion OB1 association (three new $\lambda$ Bootis stars found), NGC 2264 (two candidates) and the galactic field (five new $\lambda$ Bootis stars). Additional observations in NGC 2232 (age $\approx 210^{7} \mathrm{yr}$; Mermilliod 1981) and NGC 2301 (age $\approx 10^{8} \mathrm{yr}$; Mermilliod 1981) yielded a null result. We have been able to confirm a number of the candidate members proposed in Paunzen et al. (1997). 


\section{Photometric selection of candidate stars}

When we started this project, there was considerable confusion in the literature over which stars should be considered as members of the $\lambda$ Bootis group. A small sample of twenty well established members (Gray \& Corbally 1993) gave a general indication of the region the $\lambda$ Bootis stars occupied in the various photometric diagrams, but the boundaries of these regions were ill-defined. Since these stars are quite rare (they comprise about $1 \%$ of the field A-type stars), the main problem was to define photometric boxes which would allow the detection of $\lambda$ Bootis stars with a high probability. These boxes had to be large enough to include the whole range of possible $\lambda$ Bootis stars, but also small enough to make a spectroscopic survey feasible. We finally adopted the relations given in Table 1 as our "search box" to select candidates from their colours in the Strömgren photometric system. We have found it impractical to use derived indices such as $\delta m_{1}$ and $\delta c_{1}$ because of their insensitivity to the characteristics of $\lambda$ Bootis stars. This is especially true for stars in the main sequence band (Fig. 1). As an example we take $\lambda$ Bootis itself. The calibrations taken from Hilditch et al. (1983) give $E(b-y)=0$ and $\delta m_{1}=0.012(8)$ which implies a solar abundance for $\lambda$ Bootis, however, Venn \& Lambert (1990) have found $\log \left(\mathrm{Fe}_{\lambda \text { Boo }} / \mathrm{Fe}_{\odot}\right)=-2$. The Hauck \& Mermilliod (1990) catalogue was used as the database for our search. To avoid duplication of effort, we have removed from our candidate list stars already classified in Corbally (1984) and Gray \& Garrison (1987, 1989a,b) as well as known MK standards. In addition we have searched for possible candidates in open clusters using the list of North (1993). Members of these clusters were dereddened and checked for their location in the photometric boxes. In total a list of 600 possible candidates was compiled for the northern and southern hemisphere. Up to now about 300 stars have been observed and 120 have been classified.

\section{Survey strategy}

Our list of candidates contains stars with $5<\operatorname{mag}(V)<$ 13 in the southern as well as in the northern hemisphere. These stars must be observed spectroscopically in the blue-violet region of the spectrum in order to derive a classification on the MK system. Taking the main classification criteria for A to F-type stars (Gray \& Garrison 1987, 1989a,b) into account, the following spectroscopic requirements for our observations were chosen:

$-1500<$ resolution $\left(\frac{\lambda}{\Delta \lambda}\right)<5500$

$-S / N>150$

$-3700 \AA<\lambda_{\text {obs }}<5000 \AA$.

Resolutions lower than 1500 make it difficult to detect the characteristics of the $\lambda$ Bootis phenomenon. The broad hydrogen-line profiles (a key feature of a $\lambda$ Bootis spectrum) are difficult to normalize properly without a good expanse of continuum on either side of the line, and this led us to limit our highest resolution to 5500 .

Table 1. Selection criteria for candidates

\begin{tabular}{|c|c|c|c|c|}
\hline-0.025 & $<$ & $b-y$ & $<$ & 0.3 \\
\hline $0.13-0.3(b-y)$ & $<$ & $m_{1}$ & $<$ & $0.22-0.3(b-y)$ \\
\hline & & $c_{1}$ & $<$ & $1.4-2(b-y)$ \\
\hline
\end{tabular}

\section{Observations and reductions}

We have obtained spectra with $S / N>150$ of candidate stars selected as described in Sect. 2 at two different sites. First, two observing runs at the Observatoire de Haute-Provence (OHP) on the $1.93 \mathrm{~m}$ telescope (observer: E. Paunzen) in February/March 1994 and February 1995 were dedicated to this project. Using the CARELEC spectrograph (Lemaitre et al. 1990) with the 600 lines $\mathrm{mm}^{-1}$ grating (centered at $4200 \AA$ ) resulted in a nominal resolution of $1.8 \AA \mathrm{pixel}^{-1}$ and a spectral coverage of about $900 \AA$. Follow-up observations of candidate stars were made at the Dark Sky Observatory $0.8 \mathrm{~m}$ reflector (Appalachian State University, observer: R.O. Gray) using the Gray/Miller spectrograph with the 1200 lines $\mathrm{mm}^{-1}$ grating (blazed at $4200 \AA$ ), giving a resolution of $0.85 \AA$ pixel $^{-1}$ and a spectral coverage of about $800 \AA$. On both systems we have obtained spectra of many MK standards (Gray \& Garrison 1987, 1989a,b), some CP stars (Renson et al. 1991) and a number of confirmed $\lambda$ Bootis stars (Gray \& Corbally 1993; Paunzen et al. 1997).

All spectra were reduced using standard IRAF-routines. After correcting for the bias, flat-field and thermal background, we have checked the spectra for possible moonlight contamination. If necessary, we have used a part of each frame to correct for moonlight contamination, before applying a normalization and wavelength calibration.

\section{Classification procedure}

All programme stars were classified in the framework of a refined MK system described in Gray \& Garrison (1987, $1989 \mathrm{a}, \mathrm{b})$. In addition we used CP stars and confirmed $\lambda$ Bootis stars to guide us in the identification of peculiarities.

We classified the stars using the standard techniques of MK classification although one of us (EP) used a simple mathematical algorithm to help decide which standard star was closest to the programme star under consideration. This was done by dividing the spectra into several regions (e.g. $\mathrm{H} \gamma, \mathrm{H} \delta$, Ca II K, etc.) and the standard deviation with respect to a given MK standard for each region was calculated resulting in a first estimation 


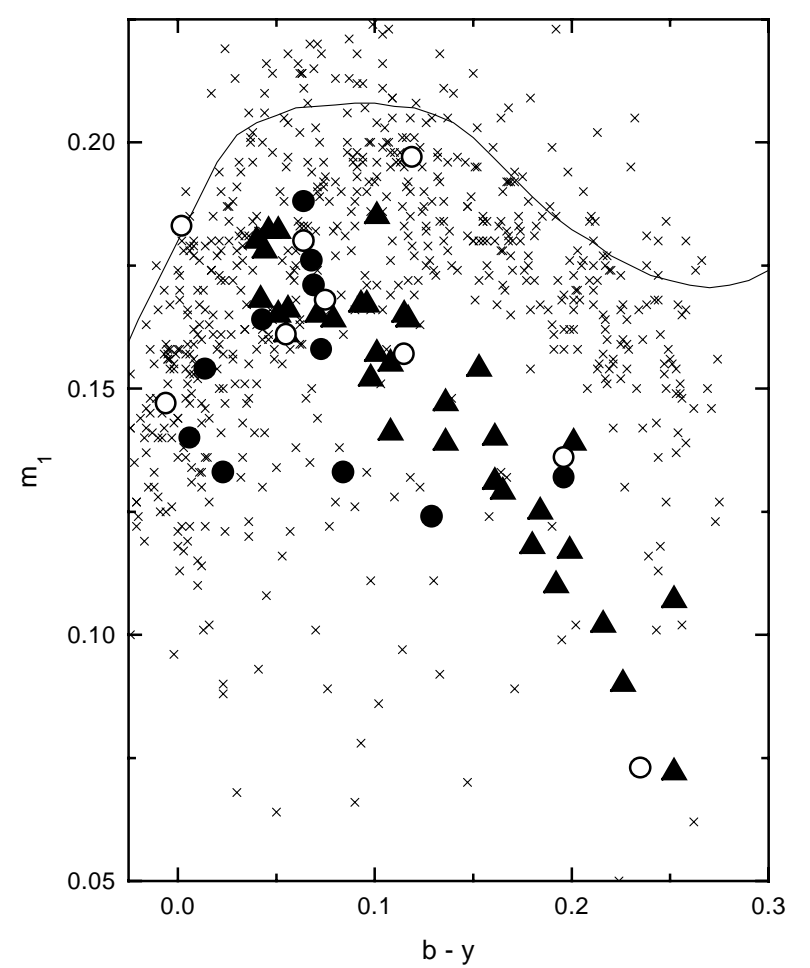

Fig. 1. $m_{1}$ versus $b-y$. Crosses are normal stars from Gray \& Garrison (1987, 1989a,b), filled triangles are $\lambda$ Bootis stars from Paunzen et al. (1997), filled circles are $\lambda$ Bootis stars discussed in Sect. 6.1, open circles are candidate $\lambda$ Bootis stars discussed in Sect. 6.2. The standard line is taken from Philip \& Egret (1980)

( \pm 2 subclasses $)$ of the spectral type. For a precise classification of the star as well as identification of possible peculiarities, the spectra were compared visually with the MK standards using hardcopies (EP) or on the computer graphics screen (ROG).

The most recent spectroscopic criteria for membership in the $\lambda$ Bootis group are given in Sect. 2.1 in Paunzen et al. (1997) and Gray (1997). Special care was taken in classifying high $v \sin i$ and probable intermediate Population II stars (Gray 1988, 1989).

In Sect. 6 all confirmed and candidate $\lambda$ Bootis stars are presented, results on "normal" stars from this sample will be presented elsewhere (Paunzen \& Heiter 1997).

\section{Results}

We have divided this section into two parts; in the first part we present our newly discovered and confirmed $\lambda$ Bootis stars and in the second part we discuss certain candidate $\lambda$ Bootis stars. The latter have a relatively high probability of being either bona-fide $\lambda$ Bootis or related stars, but follow-up observations (e.g. with higher resolution and/or higher $S / N$ spectroscopy) are required to confirm their spectral types.

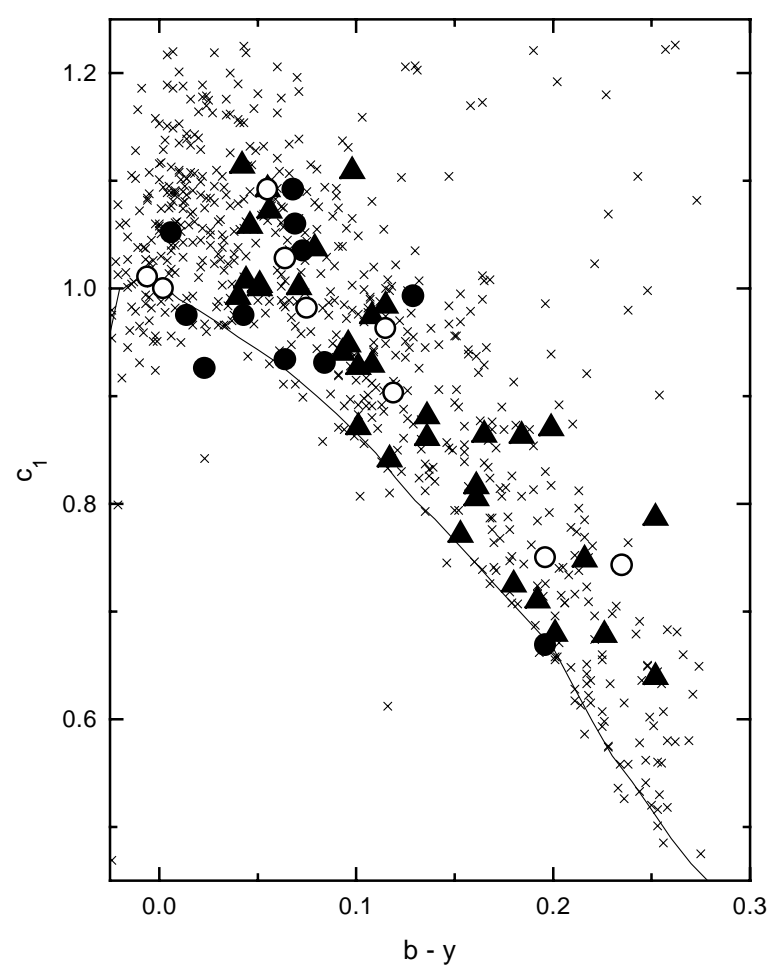

Fig. 2. $c_{1}$ versus $b-y$. Symbols are the same as in Fig. 1. Standard line is taken from Philip \& Egret (1980)

\subsection{New and confirmed $\lambda$ Bootis stars}

Based on our observational data we are able to identify six new $\lambda$ Bootis stars and confirm the membership of six stars (see Table 1 in Paunzen et al. 1997). Table 2 lists all stars with a description of their spectra. Furthermore, we have listed Strömgren photometric indices from Hauck \& Mermilliod (1990) as well as $v$ sin $i$ values from Uesugi \& Fukuda (1982) and Abt \& Morrell (1995). In the column (C) we give the classification of the hydrogen lines (normal or peculiar) according to Gray (1988). Classifications in parentheses - e.g. ( $\lambda$ Boo) indicate stars in which the $\lambda$ Bootis characteristics are mild. The last column (Status) indicates if a star is newly discovered $(\mathrm{N})$ or confirmed $(\mathrm{C})$ as $\lambda$ Bootis type.

\subsection{Candidate $\lambda$ Bootis stars}

Besides the above confirmed $\lambda$ Bootis and "normal" type stars, we have also found some interesting stars which could not be classified as $\lambda$ Bootis stars at first sight. Further observations are needed to unambiguously establish their spectral type and to avoid a misclassification. As a first step, a detailed abundance analysis is presently being carried out for three stars (HD 84123, HD 84948 and HD 101108A). The italicized spectral types are derived from our spectra. 
Table 2. New and confirmed $\lambda$ Bootis stars

\begin{tabular}{|c|c|c|c|c|c|c|c|c|c|c|c|}
\hline $\mathrm{HD}$ & HR & $\mathrm{ADS}$ & Spectral Type & $V$ & $b-y$ & $m_{1}$ & $c_{1}$ & $\beta$ & $v \sin i$ & $\mathrm{C}$ & Status \\
\hline 23392 & & & $\mathrm{~A} 0 \mathrm{Va}^{-}(\lambda \mathrm{Boo})$ & 8.70 & 0.014 & 0.154 & 0.975 & 2.917 & & NHL & $\mathrm{N}$ \\
\hline 36726 & & 4156 & $\mathrm{kA} 0 \mathrm{hA} 5 \mathrm{~mA} 0 \mathrm{~V} \lambda$ Boo & 8.81 & 0.043 & 0.164 & 0.975 & 2.922 & & NHL & $\mathrm{N}$ \\
\hline 64491 & 3083 & & $\mathrm{kA} 3 \mathrm{hF} 0 \mathrm{~mA} 3 \mathrm{~V} \lambda$ Boo & 6.23 & 0.196 & 0.132 & 0.669 & 2.734 & $\begin{array}{l}75^{1} \\
15^{2}\end{array}$ & (PHL) & $\mathrm{C}$ \\
\hline 74873 & 3481 & & $\mathrm{kA} 0.5 \mathrm{hA} 5 \mathrm{~mA} 0.5 \mathrm{~V} \lambda \mathrm{Boo}$ & 5.87 & 0.064 & 0.188 & 0.934 & 2.890 & $\begin{array}{l}85^{1} \\
10^{2}\end{array}$ & NHL & $\mathrm{N}$ \\
\hline 90821 & & & $\mathrm{kA} 2 \mathrm{hA} 7 \mathrm{~mA} 2 \mathrm{Vn} \lambda$ Boo & 9.20 & 0.068 & 0.176 & 1.092 & 2.865 & & NHL & $\mathrm{N}$ \\
\hline 91130 & 4124 & 7813 & $\mathrm{~A} 0 \mathrm{Va}^{-} \lambda \mathrm{Boo}$ & 5.93 & 0.073 & 0.158 & 1.035 & 2.854 & $\begin{array}{l}130^{1} \\
190^{2}\end{array}$ & (PHL) & $\mathrm{C}$ \\
\hline 105058 & & & kA1hA7mA1 V $\lambda$ Boo & 8.88 & 0.129 & 0.124 & 0.993 & & $130^{1}$ & (PHL) & $\mathrm{C}$ \\
\hline 120500 & & & $\mathrm{kA} 1.5 \mathrm{hA} 5 \mathrm{~mA} 1.5 \mathrm{~V}(\lambda$ Boo $)$ & 6.59 & 0.069 & 0.171 & 1.060 & 2.871 & & NHL & $\mathrm{N}$ \\
\hline 170680 & 6944 & 11411 & $\mathrm{~A} 0 \operatorname{Van}(\lambda \mathrm{Boo})$ & 5.14 & 0.006 & 0.140 & 1.052 & 2.892 & $\begin{array}{l}305^{1} \\
200^{2}\end{array}$ & NHL & $\mathrm{C}$ \\
\hline 171948 & & 11498 & $\mathrm{~A} 0 \mathrm{Vb} \lambda \mathrm{Boo}$ & 6.71 & & & & & & NHL & $\mathrm{C}$ \\
\hline 290492 & & 4211 & $\mathrm{~A} 0.5 \mathrm{Vb}(\lambda \mathrm{Boo})$ & $9.27 \mathrm{D}$ & 0.084 & 0.133 & 0.931 & 2.851 & & NHL & $\mathrm{N}$ \\
\hline 294253 & & & B9.5 Va $(\lambda$ Boo $)$ & 9.69 & 0.023 & 0.133 & 0.926 & 2.904 & & NHL & $\mathrm{C}$ \\
\hline
\end{tabular}

$v \sin i:{ }^{1}$ Uesugi \& Fukuda (1982), ${ }^{2}$ Abt \& Morrell (1995).

HD 23392: Mild $\lambda$ Bootis star, hydrogen lines show broad wings.

HD 36726: Spectrum very similar to HD 31295 (see Gray 1988), member of the Orion OB1 association, classified as A0 V (Warren \& Hesser 1978) and as Am (kA1hA1mA5) by Abt \& Levato (1977).

HD 64491: Cool $\lambda$ Bootis star similar to HD 142703 with peculiar hydrogen lines, classified as A9 Vp $(\lambda$ Boo $)$ by Abt \& Morrell (1995).

HD 74873: Classified as A1 Vp (wk 4481) by Abt \& Morrell (1995).

HD 90821: Normal hydrogen lines.

HD 91130: Shallow and broad hydrogen lines, classified as A0 Vp ( $\lambda$ Boo) by Abt \& Morrell (1995).

HD 105058: This star was first classified as A2pec ( $\lambda$ Boo) by Slettebak et al. (1968). Observations in the UV confirmed the $\lambda$ Bootis character (Faraggiana et al. 1990). Spectrum shows a very weak $4481 \AA$ line with slightly peculiar hydrogen lines.

HD 120500: Mild $\lambda$ Bootis star.

HD 170680: Classified as A0 Vp (Ca, Mg wk) by Abt (1984b), confirmed as $\lambda$ Bootis stars in the UV by Baschek \& Slettebak (1988).

HD 171948: Classified as A0 Vp ( $\lambda$ Boo) by Abt (1985).

HD 290492: Mild $\lambda$ Bootis character, star belongs to the Orion OB1 belt (Guetter 1981), classified therein as A0 V. Close binary system $\left(\Delta m=0.9, d=2^{\prime \prime}\right)$ which was resolved without any contamination.

HD 294253: Classified as $\lambda$ Bootis star by Levato et al. (1994), member of the Orion OB1 association.

It is one of the hottest as well as youngest $\lambda$ Bootis star.

HD 23258: $A 0 \mathrm{Vb}$ (sl wk metals), classified as A0 Vp ( $\lambda$ Boo) by Abt \& Morrell (1995). This star is a borderline case: the metallic line spectrum is only marginally weak, the hydrogen lines are very broad.

HD 39421: $A 1 \mathrm{Va}$ (wk 4481), classified as $\mathrm{A} 2 \mathrm{Vp}$ (wk 4481) by Abt \& Morrell (1995).

HD 66684: B9.5 Va (sl wk metals), classified as B9.5 Vp ( $\lambda$ Boo) by Abt \& Morrell (1995). Again a borderline case: Si II $4128-30$ is present and of normal strength, Mg II 4481 is only slightly weak.

HD 74911: $A 2 I V(w k$ 4481)

HD 84123 and HD 84948: Both stars show a very sim- ilar spectrum: $k A 6 h F 1 m A 6 \mathrm{~V}$ and are perhaps field horizontal branch stars. HD 84123 was found as a photometric candidate by Hauck (1986) and HD 84948 was classified as F0 V wl ( $\lambda$ Boo) by Abt (1984a).

HD 101108A: Classified as A5pec ( $\lambda$ Boo) by Slettebak et al. (1968) in the optical and was confirmed in the UV by Faraggiana et al. (1990). Our spectrum, on the other hand, result in: $A 3 I V^{-}$(wk 4481), very similar to the MK standard $\beta$ Eridani.

HD 105199: $k A 0.5 h F 0 m A 3 V \lambda$ Boo: classified as $\mathrm{A} 2 \mathrm{~V}$ by Oblak et al. (1976). This would be the first $\lambda$ Bootis star where the K-line type is in gross disagreement with the metallic-line spectrum. 
HD 149303A: $A 3 I V-V(w k$ 4481), this star is classified as $\mathrm{A} 2 \mathrm{Vp}(\lambda$ Boo) by $\mathrm{Abt}(1985)$.

Beside these galactic field stars, we have also observed stars in young open clusters. Observations in NGC 2232 and NGC 2301 yielded a null result. Two stars of NGC 2264 turned out to be good candidates for membership in the $\lambda$ Bootis group. Both stars are members of NGC 2264 according to Walker (1956).

NGC 2264 \# 87W: $A 1.5$ IV (wk 4481), hydrogen line cores are shallow, maybe due to rotation.

NGC 2264 \#138W: $A 0.5 \mathrm{~V}$ ( wk 4481), confirmed member of NGC 2264 according to Vasilevskis et al. (1965). Figures 1 and 2 show the location of all known $\lambda$ Bootis stars in the 'standard' Strömgren diagrams. Well established $\lambda$ Bootis stars were taken from Paunzen et al. (1997), normal type stars are from Gray \& Garrison (1987, 1989a,b). Although $m_{1}$ is not a good indicator of the metallicity for stars hotter than A2, the overlap (at the main sequence band, $b-y<0.1$ ) of the $\lambda$ Bootis distribution with the normal stars might be taken to suggest that there exists a continuous transition from the normal stars to the $\lambda$ Bootis stars (Fig. 1). On the other hand, it should be noted that this diagram is essentially degenerate in this region, in that even quite extreme $\lambda$ Bootis stars (such as $\lambda$ Bootis itself) are found amongst the normal A-type stars. No difference between normal dwarf and $\lambda$ Bootis stars shows up for the surface gravity in these photometric diagrams (Fig. 2).

\section{Conclusions}

We have discovered six new and confirmed six $\lambda$ Bootis stars. Furthermore, eleven candidates for membership in this group have been identified or confirmed. This yields more than ten positive detections out of 120 observed stars, showing that the proposed photometric selection criteria (see Sect. 2) in the Strömgren (as well as in the Geneva) photometric system is able to select stars with a good probability of membership in the $\lambda$ Bootis group.

With four established $\lambda$ Bootis stars (HD 36726, HD 290492, HD 290799 and HD 294253) in the Orion OB1 association $\left(10^{6}<\right.$ age $\left.<10^{7} \mathrm{yr}\right)$, two candidates (see Sect. 6.2 ) in NGC 2264 (age $\approx 510^{6} \mathrm{yr}$ ) and one candidate (HD 224964) in the Blanco 1 cluster (age $\approx 10^{7} \mathrm{yr}$ ), it is evident that $\lambda$ Bootis stars exist in young open clusters. On the other hand, the question of whether $\lambda$ Bootis stars exist in intermediate age and old open clusters (age $\gtrsim 10^{7} \mathrm{yr}$ ) remains open. Most, but not all of the A-type stars in 10 intermediate age clusters (e.g. $\alpha$ Persei, Praesepe, Pleiades, Hyades) were reclassified and no $\lambda$ Bootis candidate was found (Gray \& Corbally 1993). This seems to point to an upper limit for the $\lambda$ Bootis phenomenon $\left(10^{7} \mathrm{yr}\right)$, supporting the accretion theory. Further observations in open clusters are very much needed to establish a time scale for the $\lambda$ Bootis phenomenon.

Acknowledgements. This research was carried out within the working group Asteroseismology-AMS with funding from the Fonds zur Förderung der wissenschaftlichen Forschung (project S7303-AST). Use was made of the Simbad database, operated at CDS, Strasbourg, France.

\section{References}

Abt H.A., 1984a, ApJ 285, 247

Abt H.A., 1984b, in: Garrison R.F. (ed.) The MK Process and Stellar Classification. David Dunlap Observatory, Toronto, p. 340

Abt H.A., 1985, ApJS 59, 95

Abt H.A., Levato H., 1977, PASP 89, 797

Abt H.A., Morrell N.I., 1995, ApJS 99, 135

Baschek B., Slettebak A., 1988, A\&A 207, 112

Corbally C.J., 1984, ApJS 55, 657

Faraggiana R., Gerbaldi M., Böhm C., 1990, A\&A 235, 311

Gray R.O., 1988, AJ 95, 220

Gray R.O., 1989, AJ 98, 1049

Gray R.O., 1997, Proceedings of the Faint Blue Star conference (in press)

Gray R.O., Corbally C.J., 1993, AJ 106, 632

Gray R.O., Garrison R.F., 1987, ApJS 65, 581

Gray R.O., Garrison R.F., 1989a, ApJS 69, 301

Gray R.O., Garrison R.F., 1989b, ApJS 70, 623

Guetter H.H., 1981, AJ 86, 1057

Hauck B., 1986, A\&A 154, 349

Hauck B., Mermilliod M., 1990, A\&AS 86, 107

Hauck B., Slettebak A., 1983, A\&A 127, 231

Hilditch R.W., Hill G., Barnes J.V., 1983, MNRAS 204, 241

Jaschek C., Jaschek M., 1995, The behavior of chemical elements in stars. Cambridge University Press, Cambridge

Lemaitre G., Kohler D., Lacroix D., Meunier J.P., Vin A., 1990, A\&A 228, 546

Levato H., Malaroda S., Grosso M., Morrell N.I., 1994, PASPC 60,93

Mermilliod J.C., 1981, A\&A 97, 235

Michaud G., Charland Y., 1986, ApJ 311, 326

North P., 1993, PASPC 44, 577

Oblak E., Considère S., Chareton M., 1976, A\&AS 24, 69

Paunzen E., Heiter, U., 1997, A\&AS (in preparation)

Paunzen E., Weiss W.W., Heiter, U., North P., 1997, A\&AS 123, 93

Philip A.G., Egret D., 1980, A\&AS 40, 199

Renson P., Kobi D., North P., 1991, A\&AS 89, 61

Slettebak A., Wright R.R., Graham J.A., 1968, AJ 73, 152

Uesugi A., Fukuda I., 1982, Revised Catalogue of Stellar Rotational Velocities, Department of Astronomy, Kyoto Univ., Kyoto

Vasilevskis S., Sanders W.L., Balz A.G.A., 1965, AJ 70, 797

Venn K.A., Lambert D.L., 1990, ApJ 363, 234

Walker M.F., 1956, ApJS 2, 365

Warren W.H., Hesser J.E., 1978, ApJS 36, 497 